\title{
Dealing with Interval DEA Based on Error Propagation and Entropy: A Case Study of Energy Efficiency of Regions in China Considering Environmental Factors
}

\author{
Jianping FAN \\ School of Economics and Management, Shanxi University, Taiyuan 030006, China \\ E-mail:fjp@sxu.edu.cn \\ Weizhen YUE \\ School of Economics and Management, Shanxi University, Taiyuan 030006, China \\ E-mail:yueweizhen@sina.com \\ Meiqin WU \\ School of Economics and Management, Shanxi University, Taiyuan 030006, China \\ E-mail:wmq80@sxu.edu.cn
}

\begin{abstract}
The conventional data envelopment analysis (DEA) measures the relative efficiency of decision making units (DMUs) consuming multiple inputs to produce multiple outputs under the assumption that all the data are exact. In the real world, however, it is possible to obtain interval data rather than exact data because of various limitations, such as statistical errors and incomplete information, et al. To overcome those limitations, researchers have proposed kinds of approaches dealing with interval DEA, which either use traditional DEA models by transforming interval data into exact data or get an efficiency interval by using the bound of interval data. In contrast to the traditional approaches above, the paper deals with interval DEA by combining traditional DEA models with error propagation and entropy, uses idea of the modified cross efficiency to get the ultimate cross efficiency of DMUs in the form of error distribution and ranks DMUs using the calculated ultimate cross efficiency by directional distance index. At last we illustrate the feasibility and effectiveness of the proposed method by applying it to measure energy efficiency of regions in China considering environmental factors.
\end{abstract}

Keywords data envelopment analysis; modified cross efficiency; error propagation; entropy; energy efficiency

\section{Introduction}

Data envelopment analysis (DEA) is a non-parametric mathematical programming technique for assessing the relative efficiency of a group of decision making units (DMUs) that utilize multiple inputs to produce multiple outputs. The CCR model, introduced by Charnes et al. ${ }^{[1]}$ in 1978, is the most classical model of DEA, in which the efficiency score of a DMU is defined as the maximum of the ratio of its weighted sum of outputs to its weighted sum of inputs under the constraint that the ratio of any DMU cannot be greater than unity. The weights for

Received April 15, 2015, accepted June 29, 2015 
inputs and outputs of every DMU are the optimum solutions which maximize that efficiency itself while ignore the other DMUs' efficiency in the programming equation. A DMU is efficient if its efficiency score is equal to unity, otherwise it is inefficient. Based on self-evaluation, the CCR model, however, fails to discriminate all DMUs when the number of the efficient DMUs is more than one. To improve the discrimination power of CCR model, Sexton et al. ${ }^{[2]}$ proposed cross efficiency based on peer-evaluation, which assesses efficiency of a DMU not only in term of the optimistic efficiency (in CCR model) itself, but also its cross efficiency computed by using the weights determined by other peer DMUs. Although it has been widely used because of its strong discriminatory power, cross efficiency exist two main disadvantages, namely, non-unique weights and average aggregation assumption. Several methods have been proposed to overcome the drawback of non-unique weights, for instance, some different second goal models (such as Doyle and Green ${ }^{[3]}$, Liang et al. ${ }^{[4]}$, Wang and Chin ${ }^{[5]}$, Wang et al. ${ }^{[6]}$ ), game cross efficiency (Liang et al. ${ }^{[7]}$ ) and modified cross efficiency (Jahanshahloo et al. ${ }^{[8]}$ ). Meanwhile several cross efficiency aggregation models such as Wu et al. ${ }^{[9-10]}$, Wang and Chin ${ }^{[1]}$, Yang et al. ${ }^{[12]}$ have been proposed to avoid the assumption of average aggregation.

The entropy of a system, defined by Shannon ${ }^{[13]}$, gives a measure of uncertainty about its actual structure. It has been a useful mechanism for characterizing the information content in various modes and applications in many diverse fields. To reflect information content more overall, Liang et al. ${ }^{[14]}$ defined a new concept of entropy from the perspective of rough set theory, and the new concept of entropy was applied to determine weights of attributes by Song et al. ${ }^{[15]}$. There has not been any research on combining this new concept of entropy with DEA in previous literatures.

The traditional DEA models, such as CCR model and cross efficiency, can only deal with exact data. In the real applications, however, the interval efficiency computed by interval data can reflect the changing process of efficiency better than the exact efficiency. Several scholars have proposed some models dealing with interval data in DEA theory, and the two main models of them were introduced by Cooper et al. ${ }^{[16]}$ and Despotis et al. ${ }^{[17]}$, respectively, however the former needs complex calculation and the latter fails to measure efficiency in a unified production frontier.

Error propagation, as a method dealing with random error in physics, was combined with some multiple attribute decision making methods such as topsis method by Fan et al. ${ }^{[18]}$ and entropy by You et al. ${ }^{[19]}$ to handle interval data. There has not been any research on combining error propagation with DEA in previous literatures.

Directional distance index was proposed by Song et al. ${ }^{[15]}$ to rank alternatives completely with multiple attributes whose values are interval. In paper we use it to rank DMUs with interval efficiency completely.

Environmental issues have become one of the most important problems related with social and economic sustainable development. Evaluating energy efficiency considering environmental factors in different regions has strong theoretical and practical implications. Total-factor energy efficiency (TFEE) based on data envelopment analysis (DEA), once being introduced by $\mathrm{Hu}$ and Wang ${ }^{[20]}$, has been the most widely used method to measure energy efficiency. Related applications are $\mathrm{Hu}$ and $\mathrm{Kao}^{[21]}$, Zhang and Wang ${ }^{[22]}$, Mukherjee ${ }^{[23-24]}$, Honma and $\mathrm{Hu}^{[25]}$ and 
Yeh et al. ${ }^{[26]}$ et al. Recently, some scholars, such as Yuan et al. ${ }^{[27]}$, Bian and Yang ${ }^{[28]}$, Wang et al. ${ }^{[29-30]}$, Guo et al. ${ }^{[31]}$, Bian et al. ${ }^{[32]}$, Zhang et al. ${ }^{[33]}$, Goto et al. ${ }^{[34]}$, considered environmental factors into TFEE, which becomes a new trend to measure energy efficiency, especially that in regions in China.

The paper is organized as follows: After the introduction, Section 2 describes the method based on error propagation and entropy in interval DEA. In Section 3, the proposed method is applied to measure energy efficiency of regions in China, environmental factors considered, and the results are discussed. The conclusion is presented in Section 4.

\section{Methodology}

In this section, we introduce how to combine error propagation with DEA. The details of error propagation in multiple attribute decision making can be found in the paper written by You et al. ${ }^{[19]}$. The error distribution form of interval number $\tilde{x}=\left[x^{L}, x^{U}\right]$ is expressed as $\tilde{x}=x \pm \Delta x$, where $x=\left(x^{L}+x^{U}\right) / 2$ is the midpoint value of $\tilde{x}$ and $\Delta x=\left(x^{U}-x^{L}\right) / 2$ is the maximum error estimation of $x$.

Given function $y=f\left(x_{1}, x_{2}, \cdots, x_{m}\right)$, its propagation of errors is as follows, where its variables have the property of normal distribution.

$$
\sigma_{y}^{2}=\sum_{i=1}^{m}\left(\frac{\partial f}{\partial x_{i}}\right)^{2} \sigma_{x_{i}}^{2}
$$

where $\sigma_{y}$ and $\sigma_{x_{i}}$ is the standard deviation of $y$ and $x_{i}, i=1,2, \cdots, m$.

In practice, as we find it difficult to obtain and, it is reasonable that and are considered to be equivalent. So Equation (1) can be expressed as follows.

$$
(\Delta y)^{2}=\sum_{i=1}^{m}\left(\frac{\partial f}{\partial x_{i}}\right)^{2}\left(\Delta x_{i}\right)^{2}
$$

In paper we express inputs and outputs interval matrices in the form of error distribution. Accordingly the ultimate cross efficiency score of a DMU is calculated as interval in the form of error distribution, that is, the ultimate cross efficiency score $\tilde{\theta_{d}}$ is composed of two parts: The midpoint value $\theta_{d}$ and the maximum error estimation $\Delta \theta_{d}$. At last all DMUs can be ranked completely by directional distance index.

\subsection{Evaluation of the Midpoint Value $\theta_{d}$}

We assume that there are a set of $n$ DMUs, and each DMUj $(j=1,2, \cdots, n)$ produces $s$ outputs using $m$ inputs which are denoted as $x_{i j}(i=1,2, \cdots, m)$ and $y_{r j}(r=1,2, \cdots, s)$ respectively. For any evaluated DMUk $(k=1,2, \cdots, n)$, the efficiency score $\theta_{k k}$ can be calculated by using the following CCR model. 


$$
\begin{aligned}
\theta_{k k}=\max & \sum_{r=1}^{s} \mu_{r k} y_{r k} \\
\text { s.t. } \quad & \sum_{i=1}^{m} \nu_{i k} x_{i k}=1 \\
& \sum_{r=1}^{s} \mu_{r k} y_{r j}-\sum_{i=1}^{m} \nu_{i k} x_{i j} \leq 0, \quad j=1,2, \cdots, n \\
& \mu_{r k} \geq 0, \quad r=1,2, \cdots, s \\
& \nu_{i k} \geq 0, \quad i=1,2, \cdots, m
\end{aligned}
$$

Based on self-evaluation, the CCR model, however, fails to discriminate all DMUs when the number of the efficient DMUs is more than one. To improve the discriminatory power of DEA, cross efficiency based on peer-evaluation was proposed by Shannon ${ }^{[13]}$. The model is as follows.

$$
\theta_{k d}=\frac{\sum_{r=1}^{s} \mu_{r k} y_{r d}}{\sum_{i=1}^{m} \nu_{i k} x_{i d}}, \quad \theta_{d}=\frac{1}{n} \sum_{k=1}^{n} \theta_{k d}
$$

where $\theta_{k d}$ denotes the $d$ th DMU's cross efficiency calculated by the optimal weights of the $k$ th DMUs in CCR model.

Although it has been widely used because of its strong discriminatory power, cross efficiency exist two main disadvantages, namely, non-unique weights and average aggregation assumption.

To overcome the drawback of non-unique weights, we employ the modified cross efficiency proposed by Jahanshahloo et al. ${ }^{[8]}$ which maximizes cross efficiency and CCR efficiency simultaneously. It should be noted that modified cross efficiency can be more objective and calculated easier than other methods. The model is as follows.

$$
\begin{aligned}
\theta_{k d}=\max & \sum_{r=1}^{s} \mu_{r k} y_{r d} \\
\text { s.t. } \quad & \sum_{i=1}^{m} \nu_{i k} x_{i d}=1 \\
& \sum_{r=1}^{s} \mu_{r k} y_{r k}-\theta_{k k}^{*} \sum_{i=1}^{m} \nu_{i k} x_{i k}=0 \\
& \sum_{r=1}^{s} \mu_{r k} y_{r j}-\sum_{i=1}^{m} \nu_{i k} x_{i j} \leq 0, \quad j=1,2, \cdots, n \\
& \mu_{r k} \geq 0, \quad r=1,2, \cdots, s \\
& \nu_{i k} \geq 0, \quad i=1,2, \cdots, m
\end{aligned}
$$

where $\theta_{k k}^{*}$ is the optimum value of the $k$ th DMU in model (3).

To overcome the drawback of average aggregation assumption, we employ a new concept of entropy from the perspective of rough set theory proposed by Liang et al.[14] to aggregate the modified cross efficiency. It should be noted that the new concept of entropy can reflect information content more overall than the entropy defined by Shannon ${ }^{[13]}$. So the new concept of entropy $E_{k}$ and the entropy weight $w_{k}$ of the $k$ th evaluating DMU are defined as follows.

$$
E_{k}=\sum_{d=1}^{n} p_{k d}\left(1-p_{k d}\right)
$$




$$
w_{k}=\frac{1-E_{k}}{n-\sum_{l=1}^{n} E_{l}}
$$

where $p_{k d}=\frac{\theta_{k d}}{\sum_{l=1}^{n} \theta_{k l}}, k=1,2, \cdots, n, d=1,2, \cdots, n$.

Then $\theta_{d}$, the midpoint value of ultimate cross efficiency score of $\mathrm{DMU} d$, is calculated as follows.

$$
\theta_{d}=\sum_{k=1}^{n} w_{k} \theta_{k d}
$$

\subsection{Evaluation of the Maximum Error Estimation $\Delta \theta_{d}$}

According to $\theta_{k d}=\frac{\sum_{r=1}^{s} \mu_{r k} y_{r d}}{\sum_{i=1}^{m} \nu_{i k} x_{i d}}, \sum_{i=1}^{m} \nu_{i k} x_{i d}=1$ and Equation (2), $\Delta \theta_{k d}$ is calculated as follows.

$$
\left(\Delta \theta_{k d}\right)^{2}=\sum_{r=1}^{s} \mu_{r k}^{2}\left(\Delta y_{r d}\right)^{2}+\theta_{k d}^{* 2} \sum_{i=1}^{m} \nu_{i k}^{2}\left(\Delta x_{i d}\right)^{2}
$$

According to $\Delta \theta_{k d}, p_{k d}=\frac{\theta_{k d}}{\sum_{l=1}^{n} \theta_{k l}}$ and Equation (2), $\Delta p_{k d}$ is calculated as follows.

$$
\left(\Delta p_{k d}\right)^{2}=\frac{1}{\left(\sum_{l=1}^{n} \theta_{k l}\right)^{4}}\left[\left(\Delta \theta_{k d}\right)^{2}\left(\sum_{\substack{l=1 \\ l \neq d}}^{n} \theta_{k l}\right)^{2}+\theta_{k d}^{2}\left(\sum_{\substack{l=1 \\ l \neq d}}^{n}\left(\Delta \theta_{k l}\right)^{2}\right)\right]
$$

According to $\Delta p_{k d}$, and Equations (6) and (2), $\Delta E_{k}$ is calculated as follows.

$$
\left(\Delta E_{k}\right)^{2}=\sum_{d=1}^{n}\left(1-2 p_{k d}\right)^{2}\left(\Delta p_{k d}\right)^{2}
$$

According to $\Delta E_{k}$, and Equations (7) and (2), $\Delta w_{k}$ is calculated as follows.

$$
\left(\Delta w_{k}\right)^{2}=\frac{1}{\left(n-\sum_{l=1}^{n} E_{l}\right)^{4}}\left[\left(\Delta E_{k}\right)^{2}\left(\sum_{\substack{l=1 \\ l \neq k}}^{n} E_{l}-n+1\right)^{2}+\left(1-E_{k}\right)^{2}\left(\sum_{\substack{l=1 \\ l \neq k}}^{n}\left(\Delta E_{l}\right)^{2}\right)\right]
$$

According to $\Delta w_{k}$, and Equations (8) and (2), $\Delta \theta_{d}$ is calculated as follows.

$$
\left(\Delta \theta_{d}\right)^{2}=\sum_{k=1}^{n} \theta_{k d}^{2}\left(\Delta w_{k}\right)^{2}
$$

Then the ultimate cross efficiency score of $\mathrm{DMU}_{d} \tilde{\theta_{d}}$ is determined as $\theta_{d} \pm \Delta \theta_{d}$.

\subsection{Ranking DMUs Completely with Interval Efficiency}

Directional distance index in the form of error distribution between $\mathrm{DMU}_{d}$ and $\mathrm{DMU}_{b}$ is defined as follows to rank all $\mathrm{DMU}_{s}$ completely in term of the calculated ultimate cross efficiency score.

$$
\mathrm{DDI}\left(\mathrm{DMU}_{d}, \mathrm{DMU}_{b}\right)=\frac{1}{2}+\frac{1}{2} \frac{\theta_{d}-\theta_{b}}{\max \left(\theta_{l}+\Delta \theta_{l}\right)-\min \left(\theta_{l}-\Delta \theta_{l}\right)}, \quad l=1,2, \cdots, n
$$

So the entire directional distance index of $\mathrm{DMU}_{d}$ is expressed as follows.

$$
\mathrm{DDI}\left(\mathrm{DMU}_{d}\right)=\frac{1}{n-1} \sum_{d \neq b} D D I\left(\mathrm{DMU}_{d}, \mathrm{DMU}_{b}\right)
$$

The details of directional distance index can be found in the paper written by Song et al. ${ }^{[15]}$. 


\section{Case Study}

In this section, we provide a case study of energy efficiency of regions in China in 2010 to illustrate the comprehensive feasibility and superiority of the proposed method in Section 2.

\subsection{Model Adjustment}

Undesirable outputs are added to DEA models when we measure energy efficiency considering environmental factors. Undesirable outputs are generated when desirable outputs are produced by consuming inputs. We cannot increase desirable outputs and reduce undesirable outputs simultaneously. In order to reasonably deal with this kind of efficiency evaluation problem, undesirable outputs should be modeled based on the concept of weak disposability proposed by Färe and Grosskopf ${ }^{[35]}$.

Assume that there are a set of $n$ DMUs, and each $\mathrm{DMU}_{j}(j=1,2, \cdots, n)$ produces $s$ desirable outputs and nbad undesirable outputs using $m$ inputs which are denoted as $x_{i j}(i=$ $1,2, \cdots, m), y_{r j}^{g}(r=1,2, \cdots, s)$ and $y_{z j}^{b}(z=1,2, \cdots, n b a d)$, respectively. So model (3) and (4) can be adjusted to model (16) and (17) as follows respectively.

$$
\begin{aligned}
\theta_{k k}=\max & \left(\sum_{r=1}^{s} \mu_{r k} y_{r k}^{g}+\sum_{z=1}^{n b a d} \omega_{z k} y_{z k}^{b}\right) \\
\text { s.t. } \quad & \sum_{i=1}^{m} \nu_{i k} x_{i k}=1 \\
& \sum_{r=1}^{s} \mu_{r k} y_{r j}^{g}+\sum_{z=1}^{n b a d} \omega_{z k} y_{z j}^{b}-\sum_{i=1}^{m} \nu_{i k} x_{i j} \leq 0, \quad j=1,2, \cdots, n \\
& \mu_{r k} \geq 0, \quad r=1,2, \cdots, s \\
& \nu_{i k} \geq 0, \quad i=1,2, \cdots, m \\
& \omega_{z k}, \text { free in sign, } z=1,2, \cdots, n b a d \\
\text { s.t. } \quad & \left.\sum_{i=1}^{s} \mu_{r k} \mu_{r d}^{g}+\sum_{z=1}^{n b a d} \omega_{z k} y_{z d}^{b}\right) \\
& \sum_{r=1}^{s} \mu_{r k} y_{r k}^{g}+\sum_{z=1}^{n b a d} \omega_{z k} y_{z k}^{b}-\theta_{k k}^{*} \sum_{i=1}^{m} \nu_{i k} x_{i k}=0 \\
& \sum_{r=1}^{s} \mu_{r k} y_{r j}^{g}+\sum_{z=1}^{n b a d} \omega_{z k} y_{z j}^{b}-\sum_{i=1}^{m} \nu_{i k} x_{i j} \leq 0, \quad j=1,2, \cdots, n \\
& \mu_{r k} \geq 0, \quad r=1,2, \cdots, s \\
& \nu_{i k} \geq 0, \quad i=1,2, \cdots, m \\
& \omega_{z k}, \quad \text { free in sign, } z=1,2, \cdots, n b a d
\end{aligned}
$$

According to

$$
\theta_{k d}=\frac{\sum_{r=1}^{s} \mu_{r k} y_{r d}^{g}+\sum_{z=1}^{n b a d} \omega_{z k} y_{z d}^{b}}{\sum_{i=1}^{m} \nu_{i k} x_{i d}}, \quad \sum_{i=1}^{m} \nu_{i k} x_{i d}=1
$$


and Equation (2), Equation (9) can be adjusted to Equation (18) as follows.

$$
\left(\Delta \theta_{k d}\right)^{2}=\sum_{r=1}^{s} \mu_{r k}^{2}\left(\Delta y_{r d}^{g}\right)^{2}+\sum_{z=1}^{n b a d} \omega_{z k}^{2}\left(\Delta y_{z d}^{b}\right)^{2}+\theta_{k d}^{* 2} \sum_{i=1}^{m} \nu_{i k}^{2}\left(\Delta x_{i d}\right)^{2}
$$

\subsection{Data}

29 regions (provinces, autonomous region and municipalities) in Mainland China, excluding Chongqing, Tibet, Hong Kong, Macao and Taiwan, are selected from the perspective of data availability. GDP is taken as the desirable output, $\mathrm{CO}_{2}$ emissions as the undesirable output, capital stock, labor force and energy consumption as the three inputs. Labor force is calculated as the mean of the number of employee at the beginning and the end of the current year. Capital stock is estimated using perpetual inventory method. We estimate $\mathrm{CO}_{2}$ emissions by the product of carbon energy consumption, carbon emission coefficient and carbon conversion coefficient. All the data are collected from China Statistical Yearbook and China Energy Statistical Yearbook. Relevant descriptive statistics are summarized in Table 1.

Table 1 Descriptive statistics of inputs and outputs

\begin{tabular}{|c|c|c|c|c|c|c|}
\hline \multirow[b]{2}{*}{ Year } & \multirow[b]{2}{*}{ Statistics } & \multicolumn{3}{|c|}{ Inputs } & \multirow{2}{*}{$\begin{array}{l}\text { Desirable } \\
\text { output }\end{array}$} & \multirow{2}{*}{$\begin{array}{l}\text { Undesirable } \\
\text { output } \\
\mathrm{CO}_{2} \text { emissions } \\
\text { (Million tons) }\end{array}$} \\
\hline & & $\begin{array}{l}\text { Capital } \\
\text { stock(Billion } \\
\text { RMB) }\end{array}$ & $\begin{array}{l}\text { Labor force } \\
\text { (Thousand } \\
\text { people) }\end{array}$ & $\begin{array}{l}\text { Energy consump- } \\
\text { tion(Million tce) }\end{array}$ & & \\
\hline \multirow{5}{*}{2009} & Average & 2590.28 & 24804.78 & 120.76 & 1235.63 & 131.60 \\
\hline & St. dev. & 1853.23 & 16660.27 & 76.39 & 977.36 & 82.58 \\
\hline & Max. & 7143.39 & 58921.50 & 324.20 & 3948.26 & 348.08 \\
\hline & Min. & 316.47 & 2811.50 & 12.33 & 108.13 & 13.59 \\
\hline & Average & 3094.18 & 25458.67 & 131.60 & 1477.96 & 323.60 \\
\hline \multirow{3}{*}{2010} & St. dev. & 2213.30 & 17067.61 & 82.58 & 1143.84 & 203.07 \\
\hline & Max. & 8569.11 & 59952.00 & 348.08 & 4601.31 & 855.89 \\
\hline & Min. & 393.27 & 2898.00 & 13.59 & 135.04 & 33.40 \\
\hline
\end{tabular}

In the real applications, it is unreasonable that just regarding the statistical data at the end of the year as the indicator of an attribute because of various limitations such as statistical errors. In the paper, therefore, it should be noted that we construct data interval of each region by regarding the data at the beginning of the current year as the lower bound and the data at the end of the current year as the upper bound when we combine DEA with error propagation to measure energy interval efficiency of regions in China.

\subsection{Results and Discussion}

The results are shown in Table 2 , and the relevant discussions are as follows. 
Table 2 Efficiency results of CCR model considering environmental factors and the proposed method in Section 2

\begin{tabular}{|c|c|c|c|c|c|c|c|c|}
\hline \multirow[b]{2}{*}{$\mathrm{DMU}$} & \multicolumn{2}{|c|}{$\begin{array}{l}\text { CCR model } \\
\text { considering } \\
\text { undesirable outputs }\end{array}$} & \multicolumn{6}{|c|}{ The proposed method in Section 2} \\
\hline & $\begin{array}{l}\text { Efficiency } \\
\text { score }\end{array}$ & Ranking & $\begin{array}{l}\text { Midpoint } \\
\text { value }\end{array}$ & $\begin{array}{l}\text { Maximum } \\
\text { error } \\
\text { estimation }\end{array}$ & $\begin{array}{l}\text { Lower } \\
\text { bound }\end{array}$ & $\begin{array}{l}\text { Upper } \\
\text { bound }\end{array}$ & $\begin{array}{l}\text { DDI } \\
\text { value }\end{array}$ & Ranking \\
\hline Beijing & 1.0000 & 1 & 0.7815 & 0.4755 & 0.3060 & 1.2570 & 0.5319 & 7 \\
\hline Tianjin & 1.0000 & 1 & 0.7236 & 0.4022 & 0.3214 & 1.1258 & 0.5167 & 8 \\
\hline Hebei & 1.0000 & 1 & 0.6324 & 0.3567 & 0.2757 & 0.9891 & 0.4927 & 15 \\
\hline Shanxi & 1.0000 & 1 & 0.6631 & 0.3621 & 0.3010 & 1.0252 & 0.5007 & 13 \\
\hline Inner Mongolia & 1.0000 & 1 & 0.6193 & 0.3623 & 0.2570 & 0.9816 & 0.4892 & 16 \\
\hline Liaoning & 1.0000 & 1 & 0.6821 & 0.3569 & 0.3252 & 1.0390 & 0.5058 & 11 \\
\hline Jilin & 1.0000 & 1 & 0.5414 & 0.3479 & 0.1935 & 0.8893 & 0.4687 & 21 \\
\hline Heilongjiang & 1.0000 & 1 & 0.6856 & 0.3685 & 0.3171 & 1.0541 & 0.5067 & 10 \\
\hline Shanghai & 1.0000 & 1 & 0.9111 & 0.4941 & 0.4170 & 1.4052 & 0.5660 & 2 \\
\hline Jiangsu & 1.0000 & 1 & 0.8880 & 0.5153 & 0.3727 & 1.4033 & 0.5600 & 3 \\
\hline Zhejiang & 1.0000 & 1 & 0.8739 & 0.4498 & 0.4241 & 1.3237 & 0.5563 & 4 \\
\hline Anhui & 1.0000 & 1 & 0.4640 & 0.2825 & 0.1815 & 0.7465 & 0.4483 & 28 \\
\hline Fujian & 1.0000 & 1 & 0.6341 & 0.3386 & 0.2955 & 0.9727 & 0.4931 & 14 \\
\hline Jiangxi & 0.9793 & 28 & 0.5791 & 0.2953 & 0.2838 & 0.8744 & 0.4786 & 19 \\
\hline Shandong & 1.0000 & 1 & 0.8119 & 0.4184 & 0.3935 & 1.2303 & 0.5399 & 5 \\
\hline Henan & 1.0000 & 1 & 0.7932 & 0.3859 & 0.4073 & 1.1791 & 0.5350 & 6 \\
\hline Hubei & 1.0000 & 1 & 0.7117 & 0.3777 & 0.3340 & 1.0894 & 0.5135 & 9 \\
\hline Hunan & 1.0000 & 1 & 0.6785 & 0.3713 & 0.3072 & 1.0498 & 0.5048 & 12 \\
\hline Guangdong & 1.0000 & 1 & 1.3235 & 0.8001 & 0.5234 & 2.1236 & 0.6746 & 1 \\
\hline Guangxi & 0.9796 & 27 & 0.4848 & 0.2839 & 0.2009 & 0.7687 & 0.4538 & 26 \\
\hline Hainan & 1.0000 & 1 & 0.4853 & 0.2518 & 0.2335 & 0.7371 & 0.4539 & 25 \\
\hline Sichuan & 1.0000 & 1 & 0.6170 & 0.3447 & 0.2723 & 0.9617 & 0.4886 & 17 \\
\hline Guizhou & 1.0000 & 1 & 0.5098 & 0.2846 & 0.2252 & 0.7944 & 0.4604 & 24 \\
\hline Yunnan & 1.0000 & 1 & 0.4788 & 0.2765 & 0.2023 & 0.7553 & 0.4522 & 27 \\
\hline Shaanxi & 1.0000 & 1 & 0.5168 & 0.3038 & 0.2130 & 0.8206 & 0.4622 & 23 \\
\hline Gansu & 1.0000 & 1 & 0.5480 & 0.3130 & 0.2350 & 0.8610 & 0.4704 & 20 \\
\hline Qinghai & 1.0000 & 1 & 0.3906 & 0.2340 & 0.1566 & 0.6246 & 0.4290 & 29 \\
\hline Ningxia & 1.0000 & 1 & 0.5377 & 0.3067 & 0.2310 & 0.8444 & 0.4677 & 22 \\
\hline Xinjiang & 0.2789 & 29 & 0.5805 & 0.3874 & 0.1931 & 0.9679 & 0.4790 & 18 \\
\hline
\end{tabular}


The traditional CCR model fails to discriminate all DMUs when the number of efficient DMUs is more than 1 . When we consider environmental factors (undesirable outputs) into it, CCR model will become weaker from the perspective of discrimination power. The assumption of weak disposability of undesirable outputs can decrease production possibility set and then increase the number of efficient DMUs. As shown in Table 2, when environmental factors considered, the number of efficient regions is 26, in other words, only Jiangxi, Guangxi and Xinjiang three are inefficient.

In contrast, we use the proposed method in Section 2 to measure energy efficiency of regions in China.

First we focus on ranking results. In the latter method in Table 2, the top five most efficient regions are Guangdong, Shanghai, Jiangsu, Zhejiang and Shandong. Guangdong has the highest energy efficiency score and it ranks number 1. In 2010, The GDP value of Guangdong is 4601.30 billion RMB, performs best among all regions and is approximately 3.5 times larger than the national average level(1477.96 billion RMB), which may be the reason why Guangdong ranks number 1. Shanghai, Jiangsu, Zhejiang and Shandong follow in turn, they are all coastal regions and their economies develop fast. The bottom five most inefficient regions are Qinghai, Anhui, Yunnan, Guangxi and Hainan. Qinghai has the lowest energy efficiency score in 2010, which may be due to its less development. The GDP value of Qinghai in 2010 is 135.04 billion RMB, approximately 11 times smaller than the national average level (1477.96 billion RMB). Similarly, Anhui, Yunnan, Guangxi and Hainan are all located in the central and west area and their economy level are relatively low. To our surprise Beijing ranks 21 in the proposed method. But it may be reasonable in some way. Although it is the capital of China, Beijing as a municipality cannot contend against some provinces like Guangdong from the perspective of inputs.

Then we focus on interval efficiency. The greatest advantage of the proposed method in Section 2 is that it can show the changing process of energy efficiency of regions. As shown in Table 2, we can see the lower bound and the upper bound of energy efficiency of each region in China in 2010. We select Guangdong as an example because of its first ranking in the proposed method. The lower bound and the upper bound of energy efficiency of Guangdong are 0.5234 and 2.1236 respectively, that is, the efficiency changing scope of Guangdong in 2010 is from 0.5234 to 2.1236 .

\section{Conclusion}

In order to overcome the drawbacks of the traditional approaches dealing with interval data in DEA theory, the paper deals with interval DEA by combining traditional DEA models with error propagation and entropy, uses idea of the modified cross efficiency to get the ultimate cross efficiency of DMUs in the form of error distribution and ranks DMUs using the calculated ultimate cross efficiency by directional distance index. At last we illustrate the feasibility and effectiveness of the proposed method by applying it to measure energy efficiency of regions in China considering environmental factors.

There are two issues for further consideration. First, we can consider other DEA models such as BCC model combined with error propagation. And then, we can take into account 
other undesirable outputs, e.g., $\mathrm{SO}_{2}$, $\mathrm{NOx}$, when considering environmental factors.

\section{References}

[1] Charnes A, Cooper W W, Rhodes E. Measuring the efficiency of decision making units. European journal of operational research, 1978, 2(6): 429-444.

[2] Sexton T R, Silkman R H, Hogan A J. Data envelopment analysis: Critique and extensions. New Directions for Program Evaluation, 1986, 1986(32): 73-105.

[3] Doyle J, Green R. Efficiency and cross efficiency in DEA: Derivations, meanings and uses. Journal of the Operational Research Society, 1994, 45(5): 567-578.

[4] Liang L, Wu J, Cook W D, et al. Alternative secondary goals in DEA cross efficiency evaluation. International Journal of Production Economics, 2008, 113(2): 1025-1030.

[5] Wang Y M, Chin K S. A neutral DEA model for cross-efficiency evaluation and its extension. Expert Systems with Applications, 2010, 37(5): 3666-3675.

[6] Wang Y M, Chin K S, Luo Y. Cross-efficiency evaluation based on ideal and anti-ideal decision making units. Expert Systems with Applications, 2011, 38(8): 10312-10319.

[7] Liang L, Wu J, Cook W D, et al. The DEA game cross efficiency model and its Nash equilibrium. Operations Research, 2008, 56(5): 1278-1288.

[8] Jahanshahloo G R, Khodabakhshi M, Hosseinzadeh L F, et al. A cross-efficiency model based on superefficiency for ranking units through the TOPSIS approach and its extension to the interval case. Mathematical and Computer Modelling, 2011, 53(9): 1946-1955.

[9] Wu J, Liang L, Yang F. Determination of the weights for the ultimate cross efficiency using Shapley value in cooperative game. Expert Systems with Applications, 2009, 36(1): 872-876.

[10] Wu J, Sun J, Liang L, et al. Determination of weights for ultimate cross efficiency using Shannon entropy. Expert Systems with Applications, 2011, 38(5): 5162-5165.

[11] Wang Y M, Chin K S. The use of OWA operator weights for cross-efficiency aggregation. Omega, 2011, 39(5): 493-503.

[12] Yang G L, Yang J B, Liu W B, et al. Cross-efficiency aggregation in DEA models using the evidentialreasoning approach. European Journal of Operational Research, 2013, 231(2): 393-404.

[13] Shannon C E. The mathematical theory of communication. The Bell System Technical Journal, 1948, 27(3): 397-423.

[14] Liang J Y, Chin K S, Dang C Y, et al. A new method for measuring uncertainty and fuzziness in rough set theory. International Journal of General Systems, 2012, 31(4): 331-342.

[15] Song P, Liang J Y, Qian Y H. A two-grade approach to ranking interval data. Knowledge-Based Systems, 2012, 27: 234-244.

[16] Cooper W W, Park K S, Yu G. IDEA and AR-IDEA: Models for dealing with imprecise data in DEA. Management Science, 1999, 45(4): 597-607.

[17] Despotis D K, Smirlis Y G. Data envelopment analysis with imprecise data. European Journal of Operational Ressearch, 2002, 140(1): 24-36.

[18] Fan Z P, Guo Y J. An application of error analysis theory to the multiple attribute decision making with interval numbers. Journal of Northeastern University (Natural Science), 1997, 18(5): 555-560.

[19] You T H, Fan Z P. An error propagation method for assessing entropy weights in uncertain multiple attribute decision making. Systems Engineering, 2003, 21(1): 101-104.

[20] Hu J L, Wang S C. Total-factor energy efficiency of regions in China. Energy policy, 2006, 34(17): 32063217.

[21] Hu J, Kao C. Efficient energy-saving targets for APEC economies. Energy Policy, 2007, 35(1): $373-382$.

[22] Zhang J, Wang G. Energy saving technologies and productive efficiency in the Chinese iron and steel sector. General Information, 2008, 33(4): 525-537.

[23] Mukherjee K. Energy use efficiency in U.S. manufacturing: A nonparametric analysis. General Information, 2008, 30(1): 76-96.

[24] Mukherjee K. Energy use efficiency in the Indian manufacturing sector: An interstate analysis. General Information, 2008, 36(2): 662-672. 
[25] Honma S, Hu J. Total-factor energy productivity growth of regions in Japan. Energy Policy, 2009, 37(10): 3941-3950.

[26] Yeh T, Chen T, Lai P. A comparative study of energy utilization efficiency between Taiwan and China. Energy Policy, 2010, 38(5): 2386-2394.

[27] Yuan X L, Zhang B S, Yang W P. The total factor energy efficiency measurement of China based on environmental pollution. China Industrial Economics, 2009, 29(2): 76-86.

[28] Bian Y, Yang F. Resource and environment efficiency analysis of provinces in China: A DEA approach based on Shannon's entropy. Energy Policy, 2010, 38(4): 1909-1917.

[29] Wang K L, Yang B C, Yang L. China's provincial total-factor energy efficiency considering environmental effects. Journal of Management Science, 2010, 23(6): 100-111.

[30] Wang K L, Yang B C, Yang L. Measurement model and empirical study of China's provincial environmental efficiency of energy utilization. Systems Engineering, 2011, 29(1): 8-15.

[31] Guo X D, Zhu L, Fan Y, et al. Evaluation of potential reductions in carbon emissions in Chinese provinces based on environmental DEA. Energy Policy, 2011, 39(5): 2352-2360.

[32] Bian Y, He P, Xu H. Estimation of potential energy saving and carbon dioxide emission reduction in China based on an extended non-radial DEA approach. Energy Policy, 2013, 63: 962-971.

[33] Zhang N, Zhou P, Choi Y. Energy efficiency, $\mathrm{CO}_{2}$ emission performance and technology gaps in fossil fuel electricity generation in Korea: A meta-frontier non-radial directional distance function analysis. Energy Policy, 2013, 56: 653-662.

[34] Goto M, Otsuka A, Sueyoshi T. DEA (Data Envelopment Analysis) assessment of operational and environmental efficiencies on Japanese regional industries. Energy, 2014, 66(4): 535-549.

[35] Färe R, Grosskopf S. Modeling undesirable factors in efficiency evaluation: Comment. European Journal of Operational Research, 2004, 157: 242-245. 